\title{
Reviewers in 2019
}

(c) The Japan Society of Brain Tumor Pathology 2019

Brain Tumor Pathology Editorial Office wishes to thank the following reviewers (non-editorial board members) for their contributions in 2019.

Manabu Natsumeda

Hirokazu Takami

Kiyotaka Yokogami

Publisher's Note Springer Nature remains neutral with regard to jurisdictional claims in published maps and institutional affiliations. 